\title{
LUMINOIMMUNOMETRIC ASSAY OF TISSUE POLYPEPTIDE ANTIGEN (TPA) AND CANCER ANTIGEN 125 (CA-125) IN BREAST CANCER PATIENTS
}

\author{
O. EL-AHMADY, A.-B. HALIM AND S. EISSA* \\ Tumor Marker Oncology Research Unit, Al-A-har University and "Pathology Department, NCI, Cairo, \\ Eg.pt.
}

\section{SUMMARY}

Serum TPA and CA-125 were determined in 86 individuals ( 66 with breast cancer representing the different stages and grades of the disease and 20 normal healthy controls). TPA and CA-125 were estimated using the LIA reagents supplied by BYK Sangtec.

TPA showed sensitivity rates of $31.8 \%, 42.4 \%$ and $51.5 \%$ while CA-125 showed sensitivities of $16.3 \%, 18.6 \%$ and $25.6 \%$ at specificity levels of $100 \%, 95 \%$ and $90 \%$ respectively. Combined determination of the two markers resulted in some improvement in sensitivity. For follow-up of breast cancer patients after surgery both markers were of value and showed near-identical patterns.

KFYY WORIDS Breast cancer TPA CA- 125

\section{INTRODUCTION}

Breast cancer is the most common female malignancy of mid-life and is a leading cause of deaths among women (Omar and Contesso, 1988). Detection of localised breast cancer with negative nodes leads to $87 \%$ five years survival rate while, according to the data from the American Cancer Society (1984), if nodes are involved survival rate drops to $47 \%$ or less. No single physical technique for detecting metastasis such as radiology, ultrasound or isotope scanning proved to be $100 \%$ accurate. Attempts have been made to evaluate biological markers in blood and tissues in order to follow the course of disease during therapy, detect recurrence and possibly serve as a clue in early diagnosis (Shaarawy et al., 1990)

TPA is an unbranched peptide chain with an apparent molecular weight of $4.3 \times 10^{4}$ with an electrophoretic mobility close to that of beta-2-globulin, IP of 4.5 and sedimentation constant of $4.5 \mathrm{~S}$ (Björklund and Björklund, 1983). CA-125 is an antigenic determinant on a high-molecular-weight glycoprotein recognized by a monoclonal antibody which was raised using an ovarian cancer cell line as immunogen (Jacobs and Bast, 1989). It has been reported that TPA is the marker with the highest applicability in malignancies in general (Lüthgens and Schlegel, 1987). CA-125 has been established as a useful tool in diagnosis and monitoring of patients with ovarian carcinoma (Bast et al., 1983). It has also been evaluated in patients with non-gynecological cancer including

Correspondence to: Omar El-Ahmady, Ph.D., Head of Tumor Marker Oncology Research Unit, Al-Azhar University, 2-Roshdy Street, Safeer Square, Heliopolis, Cairo, Egypt. 
carcinoma of breast, pancreas, colon and lung but little attention has been given to its value in breast cancer (Canney et cl., 1984; Kawahara et cl., 1986). The lack of individual cancer marker tests with satisfactory sensitivity and specificity has led to investigation of the combined discriminatory power of several cancer markers (Pohl, 1992).

Luminometric assay (LIA) has been introduced recently into the field of clinical biochemistry. The availability of simple, reliable instrumentation for chemiluminescence measurement together with a wide range of assays offers a logical alternative to traditional radio-immunoassay (RIA) which has the drawbacks of short shelf-life and disposal problems (Woodhead and Weeks, 1989; Dudley, 1990).

This study was conducted to evaluate concurrent determination of CA-125 and TPA in breast cancer by the LIA technique.

\section{MATERIALS AND METHODS}

Eighty six females were included in the present study classified into two groups:

I- Breast cancer group: Sixty six females aging 30-70 years were diagnosed at National Cancer Institute (NCI) in Cairo. Histopathological examination of the tumor tissues revealed the following classes:

1- Type: Invasive ductal carcinoma (IDC) $(n=62)$, invasive lobular carcinoma (ILC) $(\mathrm{n}=2)$, intraduct papillary carcinoma $(\mathrm{n}=1)$ and adenosquamous carcinoma $(\mathrm{n}=1)$.

2- Stage: T1 $(n=2)$ TII $(n=19)$, TIII $(n=35)$ and TIV $(n=10)$.

3- Grade: G1 $(n=3)$, GII $(n=41)$, GIII $(n=22)$.

II- Normal healthy control group: Twenty females with the same range of ages as the cancer group.

Serum TPA and CA-125 were determined using the chemiluminescence reagents supplied by Byk-Sangtec Diagnostica, Germany (LIA-mat). LIA-mat assays are noncompetitive techniques using two different monoclonal antibodies: the first one is coated onto the inner surface of the test tube and the other (tracer) is covalently linked to an aminobutylethylisoluminol derivative. After the addition of standards or specimens to be analyzed to the coated tube, incubation and washing to remove unbound antigen (TPA or CA-125), the labelled antibody is added to detect a specific epitope on the antigen. After the second wash, the tube is introduced into the Optocomp I Luminometer which automatically injects alkaline peroxide solution and then the catalyst (microperoxidase) solution. On the addition of the catalyst luminol is oxidized and emits light (Dudley, 1990). The luminescence is measured for 5 seconds and the intensity is proportional to the concentration of antigen.

\section{RESULTS}

Both TPA and CA-125 were significantly elevated in the breast cancer group compared to the normal control group (Table 1), but TPA showed higher sensitivity values than CA-125 as a diagnostic tool in breast cancer. It is clear that combined determination of TPA and CA-125 improved sensitivity (Table 2). 
Table 1. Serum TPA and CA-125 in breast cancer and control groups.

\begin{tabular}{|c|cc|cc|}
\hline \multirow{2}{*}{ Markers } & \multicolumn{2}{|c|}{ TPA (U/I) } & \multicolumn{2}{c|}{ CA-125 (IU/ml) } \\
& Cancer & Control & Cancer & Control \\
& $(n=66)$ & $(n=20)$ & $(n=66)$ & $(n=20)$ \\
\hline Mean \pm SE & $* 165.5 \pm 54.9$ & $35.1 \pm 5.4$ & $* * 38.7 \pm 9.1$ & $22 \pm 1.4$ \\
Range & $37-2380$ & $8-110$ & $17.2-408$ & $9.4-49.4$ \\
\hline
\end{tabular}

* Significant difference from control group at $p=0.0() 1$.

$*$ Significant difference from control group at $\mathrm{p}=0.05$

Table 2. Cutt-off values, sensitivity rates of TPA, CA-125 and combined markers at different specificity levels.

\begin{tabular}{|ll|ccc|}
\hline \multicolumn{2}{|c|}{ Specificity \% } & $\mathbf{1 0 0}$ & $\mathbf{9 5}$ & $\mathbf{9 0}$ \\
\hline TPA: & Cut-off (U/L) & 110 & 70 & 60 \\
& Sensitivity \% & 31.8 & 42.4 & 51.5 \\
& & & & \\
CA-125: $\quad$ Cut-off (IU/ml) & 50 & 40 & 30 \\
& Sensitivity \% & 16.6 & 18.2 & 25.8 \\
& & 40.9 & 47.0 & 57.6 \\
\hline
\end{tabular}

Table 3. Serum TPA in the different stages and grades of breast cancer.

\begin{tabular}{|c|c|c|c|c|c|c|c|}
\hline \multirow[b]{2}{*}{ TPA } & \multicolumn{4}{|c|}{ Stages } & \multicolumn{3}{|c|}{ Grades } \\
\hline & $\begin{array}{c}1 \\
(n=2)\end{array}$ & $\begin{array}{c}\text { II } \\
(n=19)\end{array}$ & $\begin{array}{c}\text { III } \\
(n=35)\end{array}$ & $\begin{array}{c}\text { IV } \\
(n=10)\end{array}$ & $\begin{array}{c}I \\
(n=3)\end{array}$ & $\begin{array}{c}\text { II } \\
(n=41)\end{array}$ & $\begin{array}{c}\text { III } \\
(n=22)\end{array}$ \\
\hline Mean \pm SE (U/l) & $55.1 \pm 4.2$ & $887.1 \pm 1.3 .9$ & $222 \pm 115$ & $174 \pm 59$ & $58.8 \pm 0.4$ & $99 \pm 15.7$ & $308 \pm 208$ \\
\hline Range (U/l) & $50.9-59.2$ & $41-157$ & $36.6-2380$ & $29.7-596$ & $58.4-59.2$ & $41-252$ & $36.6-2380$ \\
\hline $\begin{array}{l}\text { Sensitivity at } \\
100 \% \text { specificity }\end{array}$ & $0 \%$ & $36.8 \%$ & $40 \%$ & $50 \%$ & $0 \%$ & $34.1 \%$ & $36 \%$ \\
\hline
\end{tabular}

$\therefore$ Signiiicant diiïerence irom $\mathrm{T} i$ at $\mathrm{p}=\hat{0} .005$.

Table 4. Serum CA-125 in the different stages and grades of breast cancer.

\begin{tabular}{|l|cccc|ccc|}
\hline \multirow{2}{*}{ CA-125 } & I & II & III & IV & I & II & III \\
& $(\mathrm{n}=2)$ & $(\mathrm{n}=19)$ & $(\mathrm{n}=35)$ & $(\mathrm{n}=10)$ & $(\mathrm{n}=3)$ & $(\mathrm{n}=41)$ & $(\mathrm{n}=22)$ \\
\hline Mean \pm SE $(\mathrm{IU} / \mathrm{ml})$ & $27.6 \pm 0.6$ & $24.6 \pm 3.3$ & $49.7 \pm 19.2$ & $3.35 .9 \pm 6.3$ & $30.5 \pm 3.5$ & $25.1 \pm 2$ & $69.9 \pm 34.2$ \\
Range (IU/ml) & $27-28.1$ & $17.2-56$ & $16.9-408$ & $19.5-7.3 .3$ & $27-34$ & $16.9-56$ & $18.3-408$ \\
$\begin{array}{l}\text { Sensitivity at } \\
100 \% \text { specilicity }\end{array}$ & $0 \%$ & $10.5 \%$ & $14.3 \%$ & $30 \%$ & $0 \%$ & $4.9 \%$ & $36 \%$ \\
\hline
\end{tabular}


No correlation was obtained between the mean values of TPA and stages of breast cancer but non-significant correlation was seen with the grades of disease. TPA exhibited high sensitivity in late stages of the disease and none of the two patients with stage I or the three patients with grade I breast cancer showed TPA above the cut-off value (Table $3)$. Although the mean values of CA-125 showed no correlation with either stages or grades of breast cancer, sensitivity rates correlated well (Table 4).

For the follow-up of breast cancer patients after surgery and during chemotherapy with cyclophosphamide, methotrexate and fluorouracil (CMF), serial determinations of TPA and CA-125 showed similar patterns (figures 1-4). It is noted that TPA changed more rapidly than CA-125. In case (2) TPA levels showed marked reduction shortly after surgery while CA-125 declined slowly. In the same case TPA increased sharply on cessation of chemotherapy but CA-125 showed no change. In case (3) TPA began to increase 4 weeks before CA-125. In case (4) TPA level decreased 2 weeks after surgery while CA-125 showed no change till the 5 th week.

\section{DISCUSSION}

The exposed site of the breast permits a diagnosis of tumor by palpation even at a tumor size of $\mathrm{T} 1$, whereas a microcarcinoma is seldom recognized exclusively by mammography. In primary breast cancer, the pre-operative cancer estimation serves for completion of the pathogram which comprises data on tumor size, lymph node involvement and receptor status (Schlegel and Lüthgens, 1989).

The present study revealed sensitivity rates of $31.8,42.4$ and $51.5 \%$ for TPA in breast cancer an specificity levels of 100,95 and $90 \%$ respectively. These sensitivity values are similar to those obtained by RIA where Kreienberg et al. (1983); Schmidt-Rode and Schulz (1984) recorded sensitivities of $45 \%$ and $40 \%$ respectively. CA-125 has been shown to be a useful tool in diagnosis and follow-up of patients with ovarian carcinoma. It showed sensitivity as high as 85\% (Björklund and Björklund, 1983; Bast et al., 1983; Kabawat et al.,1983). In breast cancer, much lower sensitivity was reported by El-Sayed et al. (1991) who found $10 \%$ and $40 \%$ positivity for tissue and serum CA-125 respectively. In the present work, CA 125 exhibited positivity rates about $50 \%$ of those for TPA. Eissa et al. in 1992 found that $48.9 \%$ of breast cancer patients have serum CA-125 above $31 \mathrm{IU} / \mathrm{ml}$.

To improve the rather low diagnostic sensitivities of single marker determination, multiple combination of biochemical markers can be used (Pohl, 1990). Our results revealed that combined determination of TPA and CA-125 was superior to the individual marker analysis. At $100 \%$ specificity we obtained a sensitivity of $40.9 \%$ by combined determination compared to $31.8 \%$ and $16.6 \%$ for TPA and CA-125 respectively.

Although the mean values of TPA or CA-125 were not found to correlate with stages or grades of breast cancer except the non-significant correlation between TPA and grades, we found that sensitivities of the markers increased as we proceed from low to high stages or grades. In 1988, El-Ahmady and Abou-Shaloc found significant correlation between TPA and stages of breast cancer. Other studies reported that rates of positivity of TPA in breast cancer gradually increase from $0-30 \%$ in stage I to 66-90\% in stage IV (Schmidt-Rhode and Schulz, 1984; Madeddu et al., 1985; Mross and Bandlow, 1986). El-Sayed et al. (1991) found no relation between either tissue CA-125 or serum CA-125 and stages or grades of breast cancer, in agreement with the findings 
TPA \& CA 125 in the follow-up of breast cancer patients after surgery
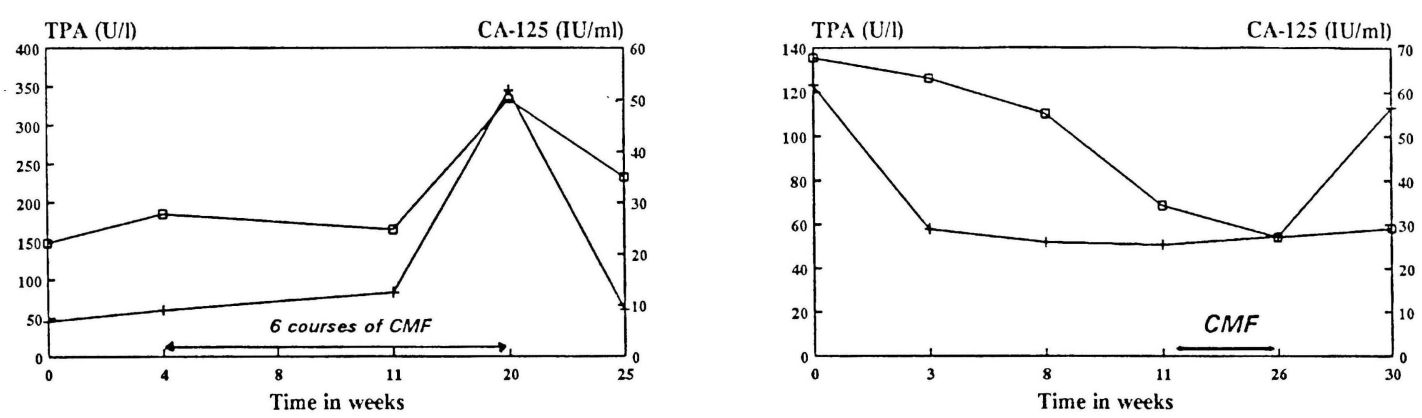

Case 2:

Case 1:

+ TPA $\rightarrow$ CA-125

$48 y$ old with IDC, T2, G3, L. N. 0

$55 \mathrm{y}$ old with IDC, T3, G3, L. N. 10/14

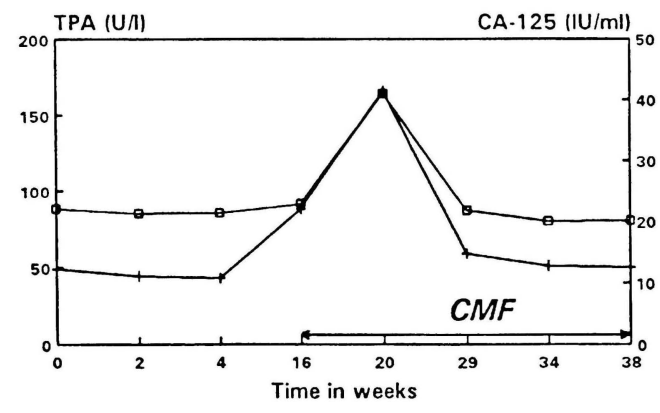

Case 3

+ TPA $\rightarrow$ CA.125

45 y old with IDC, T3, G2, L. N. $4 / 5$

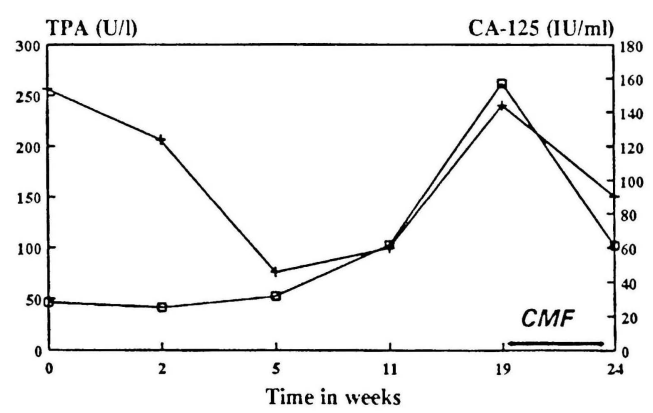

Case 4:

$+\mathrm{TPA} \rightarrow \mathrm{CA}-125$

$30 \mathrm{y}$ old with ILC, T3, G2, L. N. 10/14 
of other workers (Eissa et al., 1992). In ovarian cancer, no significant correlation was found between serum CA-125 and stage of the disease but stages II, III and IV showed sensitivity over $90 \%$ while stage I showed sensitivity of only $50 \%$ (Björklund and Björklund, 1983). Zanaboni et al. (1987) recorded elevation of serum CA-125 in 42.9\%, $93.1 \%$ and $83.3 \%$ in grades I, II and III ovarian carcinoma respectively.

Mammary carcinoma can metastasize to various regions of the body. Serum TPA shows a sensitivity of $77 \%$ for detection of metastases in various organs. Of 114 patients with recurrence of breast cancer, $49 \%$ were detected by the patients themselves and confirmed by the physicians, $40.5 \%$ were diagnosed by conventional measures such as palpation, X-ray, scintigraphy or CAT scan while the remaining $10.5 \%$ were detected only by TPA and CEA (Schlegel and Lüthgens, 1989). Nicolini et al. (1985) reported that in metastatic breast cancer patients, constant elevation or progressive increase in serum TPA and/or CEA (carcinoembryonic antigen) levels were the first pathologic findings of relapse. Shaarawy and Omar in 1989 concluded that, in general, TPA increases in progressive disease and falls again following successful therapy. It was suggested that serum CA-125 could be of potential utility in immediate post-surgical monitoring of breast cancer patients (El-Sayed et cl., 1991). An accuracy of 67\% was obtained for CA125 in the follow-up of ovarian cancer as indicated by second look laparotomy. It was stated that a persistently rising serum CA-125 level is consistently associated with progression of disease and frequently evident several months prior to clinical evidence of progression (Björklund and Björklund, 1983). Our results indicate that TPA and CA125 have similar patterns and both are in concordance with the clinical state of the patients. Elevated marker levels decrease after surgery, remain stable in clinically stable cases, begin to rise before clinical detection of metastasis and decline following successful chemotherapy. However TPA was faster in its reaction than CA-125. Bremer et al. (1992) showed that TPS (tissue polypeptide specific antigen), which is one of the specific epitope of TPA, reacts faster than cancer antigen 15-3 (CA15-3) and mammary carcinoma antigen (MCA) during monitoring of chemotherapeutic treatment of breast cancer. Other workers (van Dalen, 1992) obtained the similar findings on comparing TPS, CA 15-3 and CEA. These results were explained by the concept that TPS, as a proliferative marker, is related to tumor growth while the other markers reflect tumor mass. Rapid tumor growth is manifested before the tumor mass is affected.

In conclusion, we agree with Schlegel and Lüthgens (1989) that analysis of markers can serve as a valuable non-invasive method supporting other diagnostic measures and in surveillance of the course of the disease.

\section{REFERENCES}

Bast, R.C., Klug, T.I., St. John, E., Jenison, E., Niloff, J.M., Lazarus, H., Berkowitz, R.S., Leavitt, T., Griffiths, C.T., Parker, L., Zurawski, V.R., Knapp, R.C. (1983). A radioimmunoassay using a monoclonal antibody to monitor the course of epithelial ovarian cancer. New Engl. J. Med., 309, 883-887.

Björklund, B., Björklund, V. (1983). Specificity and basis of the tissue polypeptide antigen. Cancer Detect. \& Prevent., 6, 41-50.

Bremer, K., Richter, K., Bremer, G., Kißing, F. (1992). Clinical value of TPS in breast cancer patients. In: Kalpdor, R. (Ed). Tumor associated antigens, oncogenes, receptors, cytokines in tumor diagnosis and therapy at the beginning of nineties. W. Zuckschwerdt Verlag, München, Bern, 1992, pp. 52-56. 
Canney, P.A., Moore, M., Wilkinson, P.M., James, R.D. (1984). Ovarian cancer antigen CA-125: A prospective clinical assessment of its role as a tumor marker. Br. J. Cancer, 50, 765-769.

Dudley, R.F. (1990). Chemiluminescence immunoassay: An alternative to RIA. Laboratory Medicine, 21 (4), 216-222.

Eissa, S., Khalifa, A., Abo-Ghalia, A., Abdalla, H. (1992). Expression of CA 125 in breast cancer. In: Kalpdor, R. (Ed). Tumor associated antigens, oncogenes, receptors, cytokines in tumor diagnosis and therapy at the beginning of nineties. W Zuckschwerdt Verlag, München, Bern, 1992, pp. 499-503.

El-Ahmady, O., Abou-Shaloc, M. (1988). Predictive value of TPA and CEA in diagnosing carcinoma in breast lumps. Proc. Ist Conf. of The Egyptian Society of Tumor Markers Oncol., Feb. 15-17, 1988, Cairo, Egypt.

El-Sayed, N.K., Khalifa, A., Fadel, M.T. (1991). Tissue and serum cancer antigen 125 in benign and malignant breast lesions: A comparison with carcinoembryonic antigen. Egyptian J. Tumor Marker Oncol., 2 (2), 151-156.

Jacobs, I., Bast, R.C. (1989). The CA-125 tumor-associated antigen: A review of the literature. Human Reproduction, 4 (1), 1-12.

Kabawat, S.C., Bast, R.C., Welch, W.R., Knapp, R.C., Colvin, R.B. (1983). Immunopathologic characterization of a monoclonal antibody that recognizes common surface antigens of human ovarian tumors of serous, endometrial and clear cell types. Am. J. Clin. Pathol., 79, 98-104.

Kawahara, M., Terazaki, P.I., Chia, D., Johnson, C., Hermes, M., Tokita, K. (1986). Use of four monoclonal antibodies to detect tumor markers. Cancer, 58, 2008-2012.

Kreienberg, R., Koehler, P., Kasemyer, R., Melchert, F. (1983). Clinical utility of different tumor markers in breast cancer and gynecological malignancies. Cancer Detect. \& Prevent., 6, 221 225.

Lüthgens, M., Schlegel, G. (1987). The clinical use of tissue polypeptide antigen (TPA): A review. J. Tumor Marker Oncol., 2 (4), 261-271.

Madeddu, G., Farris, A., Casu, A.R., Arras, M.L., Campus, S. (1985). Diagnostic and prognostic value of TPA in breast cancer. Cancer Detect. \& Prev., 8, 47-52.

Mross, K., Bandlow, G. (1986). The clinical significance of tissue polypeptide antigen in breast cancer patients. Anticancer Res., 6 (2), 223-225.

Nicolini, A., Carpi, A., Giuliani, L., Di Marco, G., Giordani, R., Bianchi, R. (1985). A rational follow-up of breast cancer patients with tumor markers. 3rd European Conference on clinical oncology and cancer nursing, June 16-20, 1985, Stockholm, Sweden.

Omar, S., Contesso, G. (Eds) (1988): Breast Cancer. Korba International Ltd., London, pp. xi.

Pohl, A.L. (1990)). Tumor markers-potential limits of their application. Egyptian J. Tumor Marker Oncol., 1 (1), 7-11.

Pohl, A.L. (1992). Multiple testing with cancer markers. In: Sell, S. (Ed). Serological cancer markers. The Humana Press, Totowa, NJ, 1992, pp. 473-494.

Schlegel, G., Lüthgens, M. (1989). Breast cancer. In: Lüthgens, M., Schlegel, G. (Eds). Clinical use of the tumor marker system (CEA-TPA). Tumor Diagnostik Verlag, Leonberg, 1989, pp. 25 50 .

Schmidt-Rhode, P., Schulz, K.D. (1984). Tissue polypeptide antigen in patients with breast cancer: Longitudinal study. Prot. Biol. Fluids Proc. Colloq., 32, 731-734.

Shaarawy, M., Massoud, A.F., Milad, M. (1990). The diagnostic potential of serum CA 15.3, CEA, Katacalcin (DPN-21) and GGT in early metastatic and non-metastatic breast cancer. Egyptian J. Tumor Marker Oncol., 1 (1), 55-62.

Shaarawy, M., Omar, S. (1988). The diagnostic values of tissue polypeptide antigen (TPA), carcinoembryonic antigen (CEA) and Beta-2-microglobulin $\left(\beta_{2}-\mathrm{M}\right)$ in breast cancer. Proc. 1st Inter. Conf. of the Egyptian Society of Tumor Markers Oncol., Feb. 15-17, 1988, Cairo, Egypt. van Dalen (1992). Is TPS able to assist in decision making in breast cancer. In: Kalpdor, R. (Ed). Tumor associated antigens, oncogenes, receptors, cytokines in tumor diagnosis and therapy at the beginning of nineties. W. Zuckschwerdt Verlag, München, Bern,. 1992, pp.86-88. 
Woodhead, J.S., Weeks, I. (1989). Magic lite design and development. J. Bioluminescence and Chemiluminescence, 4, 611-614.

Zanaboni, F., Vergadoro, F., Presti, M., Gallotti, P., Lombardi, F., Bolis, G. (1987). Tumor antigen CA-125 as a marker of ovarian epithelial carcinoma. Gynecol. Oncol., 28, 61-67. 


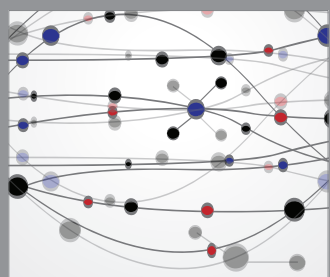

The Scientific World Journal
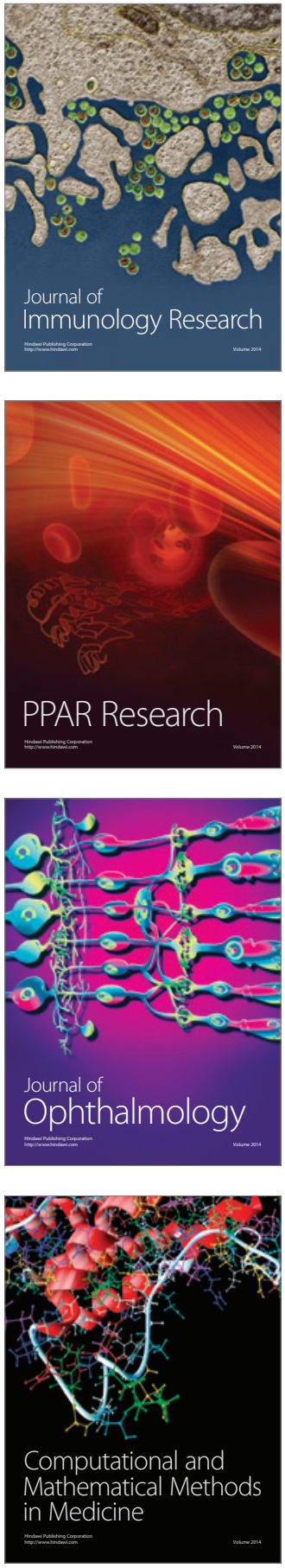

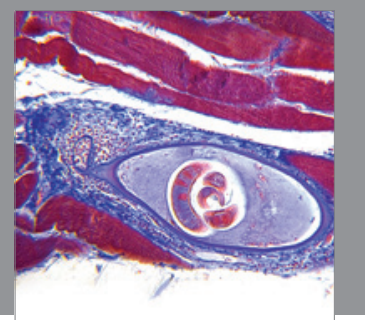

Gastroenterology

Research and Practice
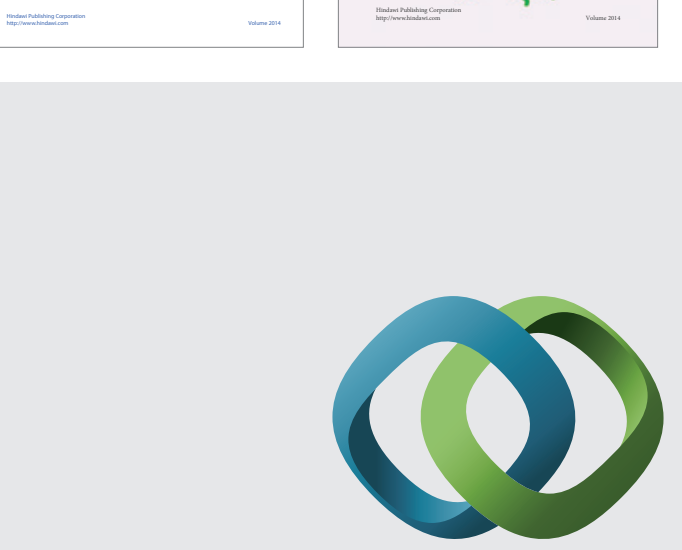

\section{Hindawi}

Submit your manuscripts at

http://www.hindawi.com
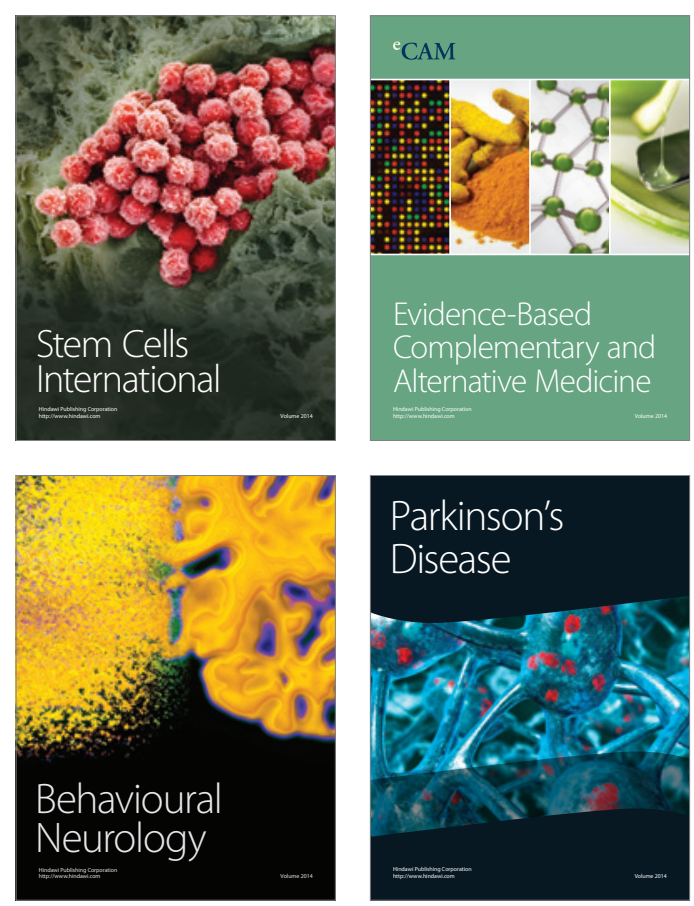

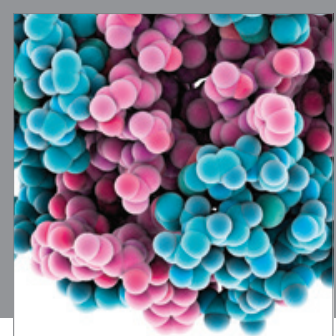

Journal of
Diabetes Research

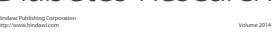

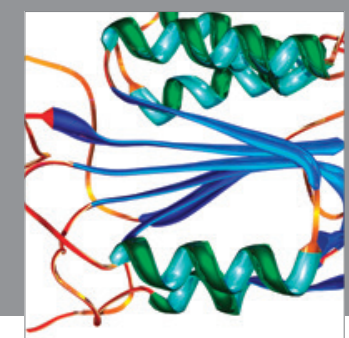

Disease Markers
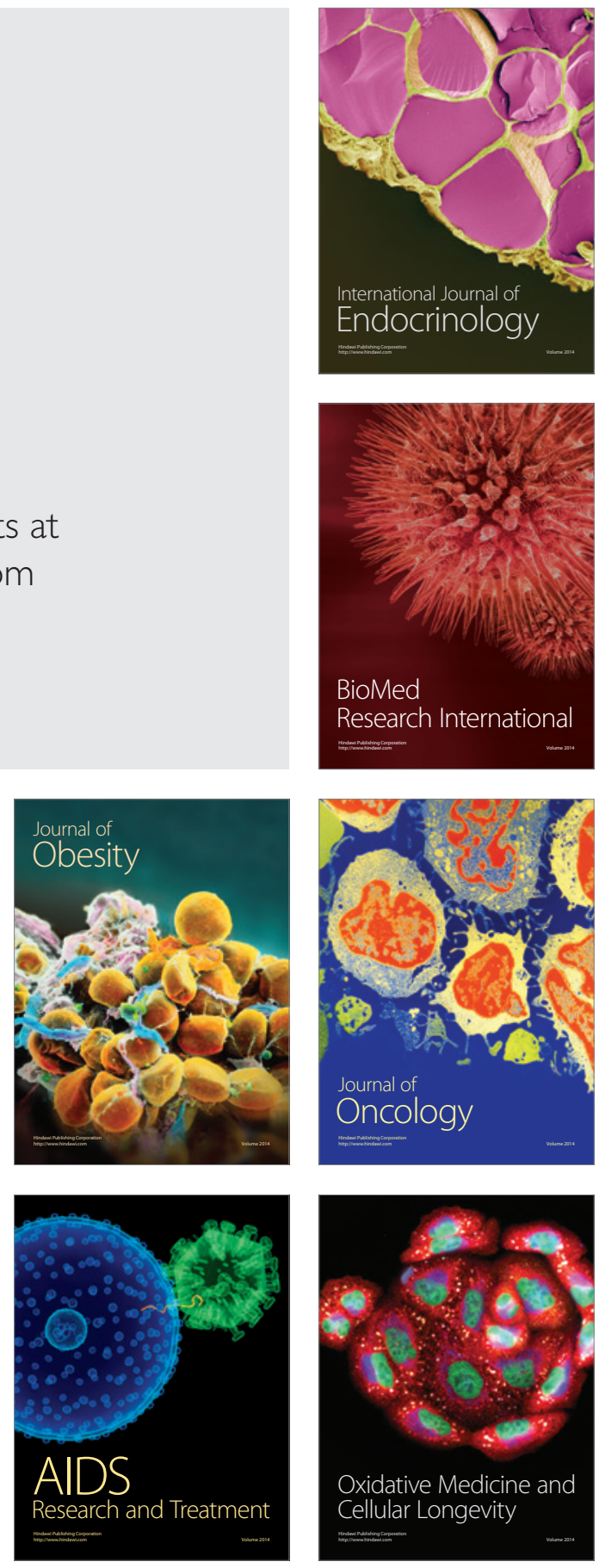